\title{
Growth and Development: a possible systemic understanding
}

\author{
GIANFRANCO MINATI \\ Italian Systems Society, Via Pellegrino Rossi 42 \\ Milan, I-20161, Italy \\ Email: gianfranco.minati@airs.it
}

\begin{abstract}

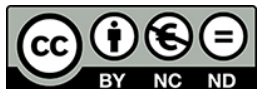

Approaches for representing quantitative processes of growth for single systems are considered focussing especially upon logistic growth processes. Attention is then extended to processes of growth for complex systems such as Multiple Systems comprising components belonging to more than one system. Complex system populations of growth processes are considered in an attempt to gain an understanding of their growth. We consider in this regard various possible approaches for understanding development and its being an emergent property. We present some possible ways of understanding emergent development which can not be suitably considered as being reducible to the properties of sequences of processes of growth.
\end{abstract}

Keywords: complex, development, emergence, growth, property.

\section{Introduction}

Processes of growth are considered here as quantitative processes with a vast range of possible representations. . We focus on the logistic process of growth since it is the more general and most widely considered in the systemic and economic literature. A more effective systemic understanding requires a consideration of generic growth processes of complex systems, such as for Multiple Systems with components belonging to more than one system and Collective Beings, particular cases of Multiple Systems where the components are autonomous agents, i.e., possessing cognitive systems allowing them to process information, as in swarms or markets. These cases involve multiple processes of quantitative growth which can be considered as a population of interacting logistic processes of growth.

Development is then considered by discussing its possible reducibility to properties of populations of growth processes. However, using a complex systems approach, development should be more properly considered as an emergent property relating, for instance, to structural dynamics and their coherence. Such an understanding of development requires the use of new conceptual approaches in contrast to those used in industrial society where the difference between growth and development is not always well defined and when, for instance, they may even be synonymous. The DYnamical uSAge of Models (DYSAM) and other approaches can be adopted for modelling and acting upon such complex systems.

In particular, the concept of the use of degrees of freedom of systems as a property may represent complex behaviour and allow actions upon the system.

The general conclusion is that for complex systems development cannot be linearly prescribed, but induced as an emergent property by considering a variety of approaches introduced in the science of complexity.

\section{The concepts of growth and development}

\subsection{Growth}

The concepts of growth and development have been elaborated in an interdisciplinary way by considering, for instance, their meaning in biology and economics (for a systemic approach see, for example, Minati and Pessa, 2006, pp. 321-334). 
Briefly, the concept of growth may be intended to relate to any quantitative process of increasing, for example:

- linear, $y=a x+b$

- factorial, $y=x$ !

- exponential, $y=e^{x}$.

Such simple processes may be suitably descriptive when considering single aspects which are easily measurable, such as financial rather than macro-economic aspects.

More sophisticated approaches are necessary for the properties of systems established by multiplicities of the same or different components interacting in different ways as in economic or biological systems.

A well-established example of such an approach is given by systems following logistic growth processes.

The so-called logistic growth following a logistic curve or logistic function was introduced by the Belgian mathematician Pierre François Verhulst (1804-1849) in the study of population growth with limited resources (Verhulst, 1990; and elaborated in Bradley, 2007 [1]; Marchetti, 1981).

By considering $N$ as the number of elements of a population having limited resources to reproduce, its growth along time $t$ is described in general by the equation $N(t)=1 / 1+e^{-t}$.

A graphical example is presented in fig. 1.

$\mathrm{N}$

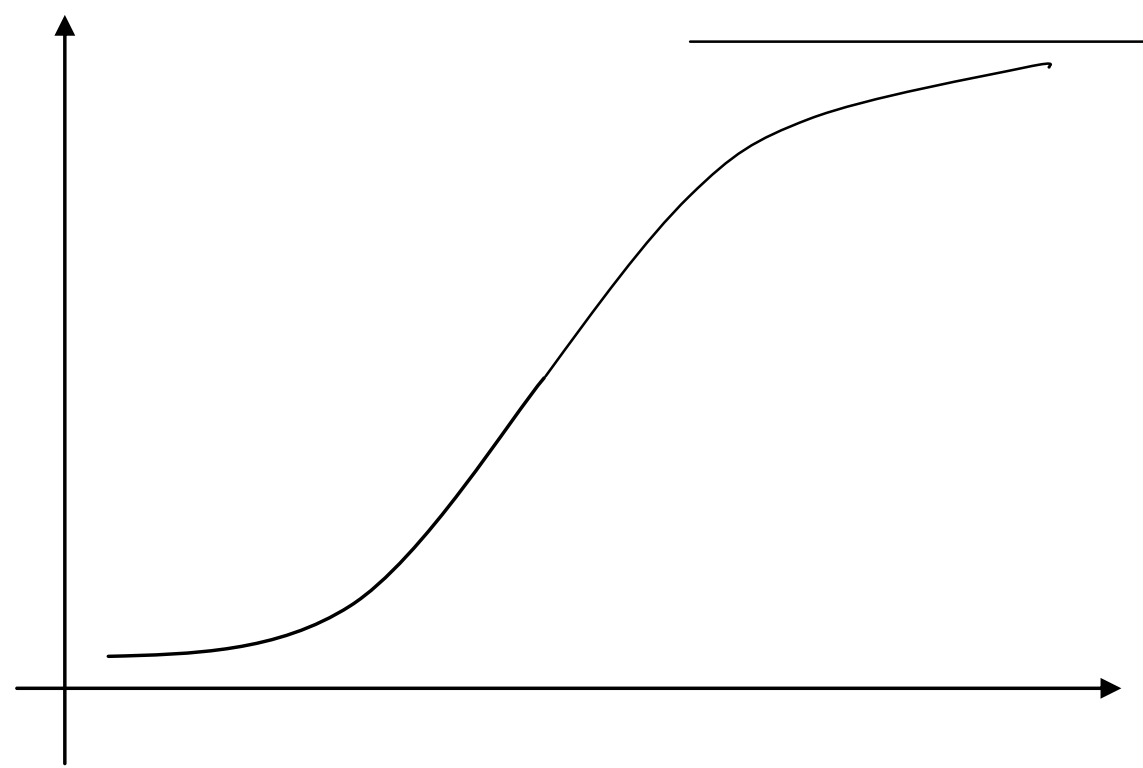

$\mathrm{t}$

Fig. 1: The logistic curve representing population growth in a context with limited resources

It should be stressed that along the logistic curve the first stage represents an increasing process of growth whereas the second stage shows a decreasing rate of increase. Moreover, the entire continuous process is limited by an asymptote. The logistic curve may be characterised in different ways by considering, for instance, the temporal duration of the first stage, the temporal duration of the second stage until it becomes asymptotical, and the value of its asymptote, i.e., the insuperable number $N$ of elements.

Such a logistic curve describes typical processes of the saturation of markets. The logistic function is applied to modelling processes of growth in various disciplinary fields such as biology and economics.

More realistically it is possible to consider, in turn, populations of processes with growth being represented by various different and possibly interacting logistic curves. One can consider a system 
of $N$ globally coupled logistic curves to study their possible mutual synchronizations during interaction (Mikhailov and Calenbuhr, 2002, p. 155).

However different approaches to modelling multiple and variable processes of growth such as quantitative increasing are possible along with their simultaneous and dynamic occurrence.

We mention how this simultaneous and dynamical occurrence may relate not only to the characteristics of quantitative increasing, but also to multiple properties as in the case of Multiple Systems and Collective Beings (Minati and Pessa, 2006, pp. 89-134).

\subsection{Multiple growths for Multiple Systems}

These concepts are recalled here since they will also be useful later when discussing the process of development.

Briefly a Multiple System (MS) can be considered as having components which simultaneously belong to more than one system. A MS may be intended as a set of systems established, or modelled as such, by the same components having multiple simultaneous or dynamic roles. When sequences of such systems are coherent and possibly changing over time they are intended as having established a MS.

Multiple simultaneous roles may be given by the fact that the state or the behaviour of an element may have different meanings such as a) the reaction of organs, considered as components of a living system, may also be a source of information when using diagnostic techniques and medical equipment; b) the output of an electronic component may also become a source of information for a security monitoring system; c) networked interacting computer systems performing cooperative tasks, as on the Internet, may have simultaneous multiple meanings.

Multiple dynamic roles may be expressed by sequences of systems such as a) virtual corporations, i.e., networked corporations establishing systems activated on demand; b) electricity networks in systems engineering (an unfortunate emergent property is the black-out) where different systems play different roles in continuously new, emerging usages.

Moreover, the study of MSs also considers interchangeability between interacting elements. In this case the coherence of a MS may be modelled by considering its emergent behaviour as ergodic occurring, in short, when, in a generic population of agents, $x \%$ of them play a specific role at any time then it is possible to assume that 'each' individual - or cluster of individuals - of the population spends $x \%$ of their total time in that role. Collective Beings (CB) are particular cases of MSs established through their agents possessing a (natural or artificial) cognitive system. In this case, their multiple roles are active, i.e., decided by the component autonomous agents considered to interact through the simultaneous or dynamic use of the same or different cognitive models. Examples are Human Social Systems when:

(a) Autonomous agents simultaneously generate and belong to different systems. For instance acting as buyers in a market and also as components of communities such as families and mobile telephone networks and traffic systems and workplaces. It means that any decision is taken by simultaneously considering such multiple belonging.

(b) Autonomous agents may dynamically give rise to various different systems, such as temporary sequential communities (e.g., communities of passengers on transport systems, queues, or an audience), at different times and without considering multiple belonging. It means that decisions are taken without considering multiple belonging.

The process of growth intended as quantitative increasing should eventually be considered for each system of MSs and CBs whereas the process of quantitative growth of MSs and CBs themselves could be suitably formulated by considering macroscopic indexes able to represent general trends allowing one to speak of growth for MSs and CBs as one does in macroeconomics regarding increases in the amount of goods and services produced over time. Such a quantitative system of increases is conventionally measured by using the Gross Domestic Product (GDP), market value of all goods and services produced over a given period of time. 


\subsection{Development}

The process of development is conceptually very different from that of growth even though they are often considered as coincident.

Within the framework of this conceptual misunderstanding, development may be considered, for instance, as given by:

- Suitable properties of growth processes such as being all positive or being positive in significant percentages;

- Establishment of new processes of increasing, which can substitute exhausted processes which are very close to their asymptotes. This is the case for sequences of consumer cycles;

- Increasing processes of growth by introducing new technologies, products and services.

This relates to the cases considered by the neoclassical or exogenous growth model of economics (see, for instance, Solow, 1956 [2]; Swan, 1956).

A less specific and more general understanding of the concept of development can be related to the emergent processes of the acquisition, maintaining and varying of positive properties by the system under study and considered to be represented by its processes of growth. More specifically, development can be considered as suitable properties of the processes of increasing intended as general processes of changing occurring within the system.

When considering processes of increasing, development may relate to the maintaining of properties such as coherence between processes given, for instance, by suitable relationships, such as their proportions and respect of their degrees of freedom, ergodicity ${ }^{1}$, sustainable consumption of resources, avoidance of contradictions and positive feedback, ability to selfregulate, and ability to substitute stability with new coherences.

This definition applies, for instance, to biological, economic and social systems.

As discussed below development relates to structural dynamics and its coherence as studied in non-linear science.

We must consider aspects of interdependence between processes of growth and development by identifying possibly necessary conditions and boundary conditions.

Development may also be intended as relating to the quantitative and structural growth of acquired evolutionary properties represented by macroscopic indexes often confused, through the adoption of a reductionist approach, with the quantitative increases of single or sets of processes of growing assumed to be necessary. Moreover, some processes may quantitatively decrease, i.e., undergo a negative increasing to sustain coherence and development.

Sustainability relates to maintaining and supporting processes which are considered as necessary, irreplaceable.

Moreover this conceptual framework excludes the appearance of processes of innovation typical of development. The concept of sustainability should be suitably reformulated with reference to development. Sustainability should be not considered as being limited to individual processes, since populations of sustainable processes may interact in such a way as to make the global system non- sustainable, but as being related to the processes of emergence themselves assumed to be positive and to be maintained. With reference to the above, the ability to develop should be intended as a property of MSs, CBs and complex systems in general where processes of emergence occur.

The ability to develop may be intended, in the science of complexity, as the ability to acquire new emergent positive structural properties, e.g., cancerous growth versus the growth of children. This issue will be examined in Section 2.

\footnotetext{
1 The concept of ergodicity is applied in economics for instance when studying the dynamics of prices (Domowitz and
} El-Gamal, 1993; 1997, [3]; 1999, [4]; Landon-Lane and Quinn, 2000, [5]). 


\subsection{DYnamical uSAge of Models (DYSAM)}

The emergent nature of development requires suitable managerial approaches. A general contextual framework is given by the science of complexity and, in case of socio-economic systems, by the ability to use new, appropriate knowledge and approaches dealing, for instance, with the shift from the industrial society to the post-industrial or knowledge society.

Approaches based on reiterating the use of the same models and concepts, merely updating a few parameters, correspond to an inability to realise that we are not playing the same game, but a variety of new different, non-equivalent games.

An example (Minati, 2012a) where this subject is elaborated is presented in Table 1.

The emergent nature of development requires multiple, eventually non-equivalent, approaches as introduced with the DYnamical uSAge of Models (DYSAM), (Minati and Brahms, 2002; Minati and Pessa, 2006, pp. 64-75) to manage the multiple properties acquired by a complex system such as a socio-economic system. This is the case when considering its development as a system of various coherent properties such as those relating to services, products, peace, health services, safety at work, and knowledge management, e.g., education, research and access to knowledge.

DYSAM is given by strategies of using independent models such as those in well-established approaches such as the Bayesian method, Ensemble Learning, Evolutionary Game Theory, Machine Learning and Pierce's abduction (Minati, 2007) while the introduction of new models is a matter of the social emergence of approaches and usages, and scientific research.

While multiple-modelling may be intended as considering the same problem from different points of view, for instance from an economic or political or legal or organizational aspect, and different problems by using specific, specialised models, DYSAM relates to networked simultaneous multi-modelling. This means the adoption of an inter-disciplinary approach when problems are reformulated in different ways such as political into economic, military into political, economical into social, and geometrical into algebraic.

DYSAM may be intended as a model of models (meta-model) considering:

- The inter-relations between different models used for the same problem;

- The multiple, simultaneous and inter-related modelling of the subsequent, acquired emergent properties.

The point of DYSAM is not to select the best model once for all, but to adopt suitable strategies to use all of them simultaneously by applying the meta-knowledge related to the mutual representations of levels of knowledge. We emphasize that this point relates to the ability to consider, and eventually introduce, a variety of approaches and to use them coherently.

\begin{tabular}{|l|l|}
\hline Concepts used in the Industrial Society & \multicolumn{1}{c|}{ Concepts used in the Post- Industrial Society } \\
\hline Completeness & Non-completeness as a resource \\
\hline Computability & Non-Computability \\
\hline $\begin{array}{l}\text { Linear correspondence between micro and } \\
\text { macro }\end{array}$ & Non-linear correspondence between micro and macro \\
\hline Deciding as optimisation & Process of deciding as emergent \\
\hline Equilibrium & Coherence \\
\hline Measure & Properties of multiple measurements \\
\hline Multiplicity as set & Multiplicity as system \\
\hline Optimise & Generate coherence \\
\hline Property possessed & Properties acquired \\
\hline Reversibility-irreversibility & Non-reversibility as a source of uniqueness \\
\hline Solve & To manage using multiple approaches \\
\hline Stability & Coherent Dynamics \\
\hline
\end{tabular}

Tab. 1: Example of the contrast between concepts used in the Industrial and Post-Industrial Society 


\section{Systemic understanding}

In Section 1 the concepts of growth and development were presented. The systemic understandings focussed upon here relate to the multiple processes of growing occurring in complex systems as represented through the use of MSs and CBs. Development can be considered as being given and represented by, or being related to, processes of growth and suitable indexes appropriate for representing their combinations and relationships.

We are considering here development as emergent and not additive or even given by nonlinear combinations of indexes of growth.

Development should be considered conceptually as being a part of systemic emergent properties such as life and health for living systems; behaviour of collective systems such as flocks, swarms and traffic; properties of networks such as the Internet and electricity networks.

Such is the nature of development which requires suitable approaches developed and used in the science of complexity, such as DYSAM.

Another approach is given by the science of networks when modelling a socio-economic system such as a network (see, for instance Barabási, 2002; Dorogovtsev, et. al., 2008; Lewis, 2009; Motter and Réka, 2012).

Another possibility is given by considering development as an emergent property given by structural changing or acquired through structural dynamics as considered by meta-structures for modelling processes of emergence and the acquisition of properties (Minati, G., 2008, [7]; Minati, 2012b; Minati, 2012c; Minati and Licata, 2012; Pessa, 2012). The dynamics considered concern sequences of different structures as already dealt with in the literature for some aspects of Neural Networks and multi-level hypernetwork dynamics of complex systems. This is in contrast with considering the dynamics of systems as possessing a unique structure where elements interact by using the same and fixed interaction structures of rules. A more effective understanding requires concepts and approaches used in the science of complexity, see Table 2. 


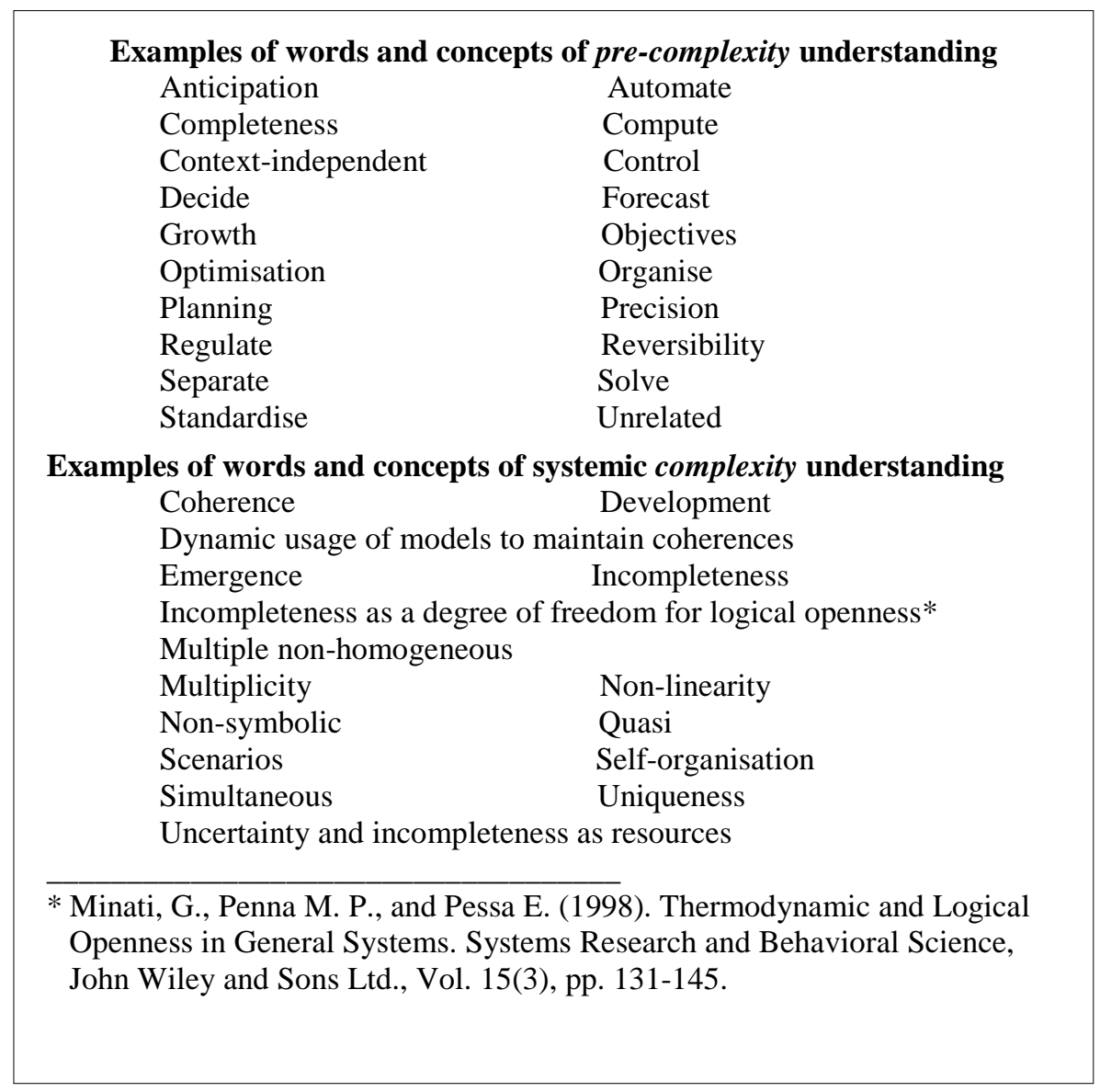

Tab. 2: Words and concepts from pre-complexity and complexity

The theoretical constructivist role of the observer must be stressed when inventing rather than selecting the level of description such as the mesoscopic variables in the metastructural approach (Licata and Minati, 2010, [8]).

In conclusion, development should be intended as an emergent property not to be decided, regulated, prescribed, and facilitated, but rather induced by using models for complexity.

\section{Interdependence between growth and development}

Important questions relate to the possible interdependence of multiple quantitative processes of growth and development. For instance:

- Is growth a necessary condition for development?

- Which relationships exist between multiple quantitative processes of growth and development? Are there correspondences between multiple quantitative processes and possible consequent multiple developments?

- Can multiple quantitative processes even of negative growth lead to balancing effects so as to acquire coherence and then lead to development?

- Consumer product life-cycle sequences induced by partial innovations may move up to the asymptote of growth curves. What will be their effects on the strategy for inducing the emergence of development?

- Is it possible to identify boundary conditions for multiple quantitative processes of growth related to eventual collapse of the system?

- How are structural dynamics and their coherence(s) related to multiple quantitative processes of growth? 
Such questions are examples of issues to deal with when formulating scenarios and strategies for complex systems. Biological complexity is a source of several such approaches (Kauffman, 1993; Luisi, 1996).

\section{Modifying actions}

After having introduced the concepts above, we now consider the general problem for complex systems in developing models and approaches suitable for recognising the emergence of collective behaviours even where there is no recognition of acquired properties for any reason such as problems of scaling, and for adopting non-catastrophic interventions able to:

a) induce coherence within sets of elements interacting collectively but without constituting a complex system or giving rise to processes of emergence (such as Brownian motion, crowds or populations of protocells);

b) vary, change, combine and maintain collective behaviours;

c) keep or restore coherence of a collective behaviour in the face of possible changes or a loss of coherence;

d) destroy or prevent the establishment of coherence and related processes of emergence to be avoided for any reason.

This is the case when considering socio-economic systems and the property of development considered as emerging from populations of interacting agents or networks of nodes (representing, for instance, buyers, users of services, and corporations) or multiple processes of growing (representing, for instance, markets, usage of services, and financial trends). Suitable disciplinary levels of description should be used at microscopic, macroscopic and mesoscopic levels.

In the literature on complex systems, there is an inability of several interdisciplinary models introduced, e.g., in physics, biology and economics, to allow research not only to simulate, but to change and maintain properties acquired by collective behaviours.

Research is now looking for approaches and models able to allow interventions not for explicitly prescribing, i.e., by using analytical representations, the dynamics of the model itself, parametrical changes and assuming independence and separability from the environment, but rather inducing the acquisition of emergent properties.

Extended classical approaches may consider networked, non-linear, multiple, variable, simultaneous and subsequent causations still focusing on the strategy of looking for the best model and learning by assuming stability, replicability, and adaptation as valuable aspects.

Examples of different approaches introduced in the literature of the science of complexity include those considered by:

a) Network Science by acting, for instance, on the topology of networks and the fitness of nodes;

b) DYSAM;

c) MSs and CBs;

d) Regulating boundary conditions and their changes to obtain necessary resources such as those relating to energy being made available, or to financial, temporal and spatial structures;

e) Dynamical structures represented by meta-structural properties;

f) Power laws ${ }^{2}$;

2 If the frequency of a phenomenon varies as a function of one of its attributes, e.g., dimension or number of nodes, then it is said to follow a power law. For instance the number of cities varies with the size of their population. A general power law is $f(x)=x^{k}$. Consider the example above when

- $x$ refers to the size of the population,

- $f$ assigns the number of cities possessing that population,

- $\quad k$ is a constant,

the scale invariance of such a power law is given by the fact that if $x$ scales for a parameter $q$ we have 
g) Scale free ${ }^{3}$;

h) Properties of attractors in chaotic behaviour;

i) Dynamical symmetries and quasi-periodicity;

j) Any combination of the above a-i;

k) Quantum-based models.

An interesting example of a new conceptual approach is given by not limiting oneself to prescribing the degrees of freedom for systems, but to consider what happens between the degrees of freedom, how the system uses them and how to induce their usage. The assumption is that complexity emerges between degrees of freedom and through their usage. In order to clarify this case let us consider a collective system such as a swarm or a market established by $k$ interacting agents. By considering a behavioural aspect $A_{k}$, such as speed, value of a financial transaction, amount of credit applications and number of workers, the following degrees of freedom can be considered as valid for each of the $k$-interacting agents, given that the value of $A_{k}$ must be not greater than $A_{\max }$ nor less than $A_{\min }$.

Consider, instant by instant, $A_{k(t)}$ of each of the $k$-interacting agents. One can calculate the degree of use of the available degrees of freedom as a percentage of the difference between the maximum and minimum permissible:

$$
\left(\left[A_{k}(t)-A_{\min }\right] * 100\right) /\left[A_{\max }-A_{\min }\right] .
$$

Consider the case when $A_{\max }=50$ and $A_{\min }=5$ and their difference (50-5) $=45$.

- If the value of $A_{k}(t)$ is 15 at time $t_{1}$ it means that it is using the degree of freedom to a percentage of $([15-5] * 100 /(45]=22,2 \%$.

- If the value of $A_{k}(t)$ is 42 at time $t_{2}$ it means it is using the degree of freedom to a percentage of $([42-5] * 100 /(45]=82,22 \%$.

- If the value of $A_{k}(t)$ is 6 at time $t_{3}$ it means that it is using the degree of freedom to a percentage of $([6-5] * 100 /(45]=2,22 \%$.

Extremes occur when $A_{k}(t)$ takes the values:

- 50 and the percentage is $([50-5] * 100 /(45]=100 \%$

- 5 and the percentage is $([5-5] * 100 /(45]=0 \%$.

The point is that sequences of these percentages provide a history of use, a behavioural profile of use of the degrees of freedom on the part of each agent.

Properties of sets of these percentages, such as regular periodicity and quasi-periodicity, accumulations in specific zones or distributions, can be considered as meta-structural properties representing properties of a collective behaviour. It is also possible to consider the possibility of prescribing them through self-regulatory approaches for the agents, through action on the resources available for interacting, structures and the possible introduction of suitable perturbative, but also regulatory effects.

\section{Conclusion}

A systemic understanding of the difference between the processes of growth and development is presented. In particular, development is identified as an emergent property acquired by complex

$$
f(q x)=(q x) k=q k f(x) .
$$

This is the case in biology for the relation between metabolic rate (B) - energy expressed in watts consumed by an individual at rest in the time unit - and the body mass $(\mathrm{M})$ of living organisms. If we consider $f$ to assign the metabolic rate to individuals with mass $M$ we have $f(M)=M^{3 / 4}$ valid for living organisms of any size less than a coefficient of normalization.

3 A network is said to be scale free if the degree of distribution, i.e., the probability that a node selected at random has a certain number of links, follows a power law without depending on scalar parameters of the structure. Examples are given by the Internet, metabolic networks, the network of blood vessels and neurons. The peculiarity of these networks is that, in processes of growth, the number of links of a node depends on a power-law considering the number of existing links. 
systems where processes of growth can occur. Possible relationships and interdependence between the two processes and suitable possible approaches for acting upon acquisition, maintaining and variation of development are discussed.

The general conclusion is that development for complex systems as socio-economic systems cannot be linearly prescribed by using tools and approaches suitable for the industrial age characterised by relatively few relationships and interactions, by physical resources rather than knowledge, by fixed rather than dynamical structures, but can be induced as an emergent property by considering a variety of approaches introduced by the science of complexity.

For complex socio-economic systems classical approaches used in the non-complex industrial age are almost completely ineffective, such as looking for optimisation, precision, completeness, planning, fixed organisation, stability, replicability, and possibility to learn from the past in a context assumed to be structurally fixed. New models and approaches based on multiple causations and models, induction by using higher level properties such as topological ones, or those based on attractors, power laws and scale free properties are required not just as tools to be used within the traditional view, but to be transformed within a culture pervading entire social systems.

This requires new styles of management, new understanding of the concept of government, to renounce previous approaches and objectives as a premise for development ${ }^{4}$, and for the introduction of new economic knowledge.

\section{References}

Barabási, A. L., 2002, Linked: The New Science of Networks. Cambridge, Massachusetts, Perseus Publishing.

Domowitz, I. and El-Gamal, M., 1993, A Consistent Test of Stationary Ergodicity, Econometric Theory Vol. 9. pp. 589-601.

Dorogovtsev, S. N., Goltsev, A. V. and Mendes, J. F. F., 2008, Critical phenomena in complex networks, Reviews of Modern Physics, Vol. 80, pp. 1275-1335.

Drucker, P. F. (1970), Technology, Management \& Society. Harper \& Row, New York, NY.

Kauffman, S. A. 1993, Origins of Order: self organization and selection in evolution. Oxford University Press, NY.

Lewis, T. G., 2009, Network science: Theory and applications, Hoboken, NJ, Wiley.

Luisi, P. L., 1996, The Emergence of Life: From Chemical Origins to Synthetic Biology.

Cambridge University Press, Cambridge, UK.

Marchetti, C., 1981, Society as a learning system: Invention, and Innovation Cycles Revisited, Technological Forecasting and Social Change, Vol. 18, pp. 267-282.

Mikhailov, A. S. and Calenbuhr, V., 2002, From cells to societies. Models of complex coherent actions. Springer, Berlin.

Minati, G. , 2007, Some new theoretical issues in Systems Thinking relevant for modelling corporate learning, The Learning Organization (TLO), Vol. 14(6), pp. 480-488.

Minati, G., 2012a, Knowledge to manage the Knowledge Society, Learning Organisation, The, Vol. 19 (4), 352 - 370.

Minati, G. , 2012b, First draft of an experimental protocol for research into Meta-Structural properties in simulated collective behaviour. Research issues and possible applications. In: G.

Mined, M. Abram and E. Pessa (Eds.), Methods, Models, simulations and Approaches Towards a general theory of change. World Scientific, Singapore, pp. 95-112.

Minati, G., 2012c, Introduction to the meta-structures project: prospective applications, World Futures, Vol. 68 (8), pp. 558-574.

\footnotetext{
${ }^{4}$ The first thing to be decided in a strategy of development is what to leave out and not to identify new objectives (Drucker, 1970).
} 
Minati, G. and Brahms, S., 2002, The Dynamic Usage of Models (DYSAM). In G. Minati and E. Pessa (Eds.), Emergence in Complex Cognitive, Social and Biological Systems, Kluwer, New York, pp. 41-52.

Minati, G. and Licata, I., 2012, Meta-Structural properties in Collective Behaviours, The International Journal of General Systems Vol. 41 (3), pp. 289-311

Minati, G. and Pessa, E., 2006, Collective Beings, Springer, New York.

Motter, A. E. and Réka, A., 2012, Networks in motion, Physics Today, Vol. 65(4), pp. 43-48.

Pessa, E., 2012, On Models of Emergent Metastructures. In: G. Minati, M. Abram and E. Pessa (Eds.), Methods, Models, simulations and approaches towards a general theory of change, World Scientific, Singapore, pp. 113-134.

Solow, R. M., 1957, Technical Change and the Aggregate Production Function", Review of Economics and Statistics, Vol. 39 (3), pp. 312-320.

Swan, T. W., 1956, Economic Growth and Capital Accumulation, Economic Record, Vol. 32 (2), pp. 334-361.

Verhulst, F., 1990, Non linear differential equations and dynamical systems. Springer, Berlin.

\section{Web Resources}

[1] Bradley, D. M., 2007, Verhulst's logistic curve, Proceedings of the National Academy of Sciences (PNAS), pp. 1-5, Retrieved October 22, 2008, from http://arxiv.org/PS_cache/arxiv/pdf/0706/0706.3163v1.pdf

[2] Solow, R. M., 1956, A Contribution to the Theory of Economic Growth, Quarterly Journal of Economics, Vol. 70 (1): 65-94. http://qje.oxfordjournals.org/content/70/1/65.full.pdf+html

[3] Domowitz, I. and El-Gamal, M., 1997, Financial Market Structure and the Ergodicity of Prices, http://www.ssc.wisc.edu/econ/archive/wp9719.pdf

[4] Domowitz, I. and El-Gamal, M., 1999, A Consistent Nonparametric Test of Ergodicity for Time Series with Applications, http://papers.ssrn.com/sol3/papers.cfm?abstract_id=179912,

[5] Landon-Lane, J. S. and Quinn, J. A., 2000, Growth and Ergodicity: Has the world converged? The Econometric Society, http://www.econometricsociety.org/meetings/wc00/pdf/0146.pdf

[6] Meta-structures Project http://www.meta-structures.org/

[7] Minati, G., 2008 ,New Approaches for Modelling Emergence of Collective Phenomena. The Meta-Structures Project. Polimetrica, Milan.

http://www.polimetrica.com/?p=productsMore\&iProduct=81\&sName=new-approaches-for-modelling-emergence-ofcollective-phenomena-(gianfranco-minati)

[8] Licata, I. and Minati, G., (2010), Creativity as Cognitive design - The case of mesoscopic variables in Meta-Structures, In: Creativity: Fostering, Measuring and Contexts (Alessandra M. Corrigan, ed.), Nova Publishers, New York, pp. 95-107 http://cogprints.org/6637/1/CreativityasDesign-NOVA.pdf 
